\title{
Mobile Stress Recognition and Relaxation Support with SmartCoping: User-Adaptive Interpretation of Physiological Stress Parameters
}

\author{
Ulrich Reimer \\ University of Applied \\ Sciences, St. Gallen \\ ulrich.reimer@fhsg.ch
}

\author{
Emanuele Laurenzi \\ University of Applied \\ Sciences, St. Gallen \\ emanuele.laurenzi@fhsg.ch
}

\author{
Edith Maier \\ University of Applied \\ Sciences, St. Gallen \\ edith.maier@fhsg.ch
}

\author{
Tom Ulmer \\ University of Applied \\ Sciences, St. Gallen \\ tom.ulmer@fhsg.ch
}

\begin{abstract}
The paper describes a mobile solution for the early recognition and management of stress based on continuous monitoring of heart rate variability (HRV) and contextual data (activity, location, etc.). A central contribution is the automatic calibration of measured HRV values to perceived stress levels during an initial learning phase where the user provides feedback when prompted by the system. This is crucial as HRV varies greatly among people. A data mining component identifies recurrent stress situations so that people can develop appropriate stress avoidance and coping strategies. A biofeedback component based on breathing exercises helps users relax. The solution is being tested by healthy volunteers before conducting a clinical study with patients after alcohol detoxification.
\end{abstract}

\section{Introduction}

Stress is the body's normal response to a real or implied threat. In small doses, stress can help us perform under pressure, make us stay focused, energetic and alert. However, if stress symptoms persist, it starts causing major damage to our health, productivity, relationships and quality of life. Chronic stress can cause hypertension, suppress the immune system, increase the risk of heart attack and stroke, and make people more vulnerable to anxiety, addictive behavior and depression [20, 28]. Excessive and prolonged stress may cause burnout, a state of emotional, mental and physical exhaustion.

We cannot completely eliminate stress from our lives, but we can learn how to cope with it by controlling stress-inducing situations and physiological reactions. This, however, requires that we are aware of the fact that we are stressed at a particular moment, by certain events or by encounters with specific persons. With this in mind, the publicly funded research project SmartCoping ${ }^{1}$ set out to develop a mobile app for stress

\footnotetext{
${ }^{1}$ The project was funded by the Swiss Confederation's innovation promotion agency under grant number 14049.2 PFES-ES.
}

warning and relaxation support. The project has pursued the following main goals:

- Help users enhance their self-perception so that they are able to recognize when they suffer from stress. This is achieved by continuous monitoring of stress levels and warning users when their stress level is too high. The assumption is that after some time users no longer need this feature of the app.

- Give users hints in which situations they experience significantly higher stress so that they may develop avoidance or coping strategies.

- Help users relax with a biofeedback component. The SmartCoping app addresses two scenarios:

1. The prevention of chronic stress: The target group consists of individuals who are or feel threatened by stress or who are interested in measuring and documenting their vital as well as contextual data so as to increase their self-awareness and long-term health [38].

2. Therapeutic and rehabilitation support for conditions caused by stress: Here the target group are inor outpatients who need support in avoiding stress, e.g. patients after alcohol detoxification, burn-out patients, or patients suffering from depression. In this scenario the therapist or nurse may have access to the data if the patient agrees.

In the following section we discuss the state of the art in stress detection and management. Section 3 describes the innovative aspects of SmartCoping. Section 4 explores the possible ways of measuring stress. Section 5 is the core of the paper and presents the approaches underlying SmartCoping. Section 6 describes the evaluation being done while Section 7 concludes the paper.

\section{Stress Management: State of the Art and Application Areas}

There is a plethora of health-related apps on the market including apps for coping with stress, which give stress warnings, offer progressive muscle relaxation, 
guided exercises, etc. Whereas some apps try to determine the individual stress level by asking a series of questions, the more innovative apps use the sensors integrated in many of today's smartphones as well as external sensors to recognise and display stress symptoms and monitor them over time. An interesting approach was pursued by the Mobile Heart Health Project driven by Intel researchers. They used a wireless ECG to detect changes in stress levels as captured by heart rate variability (HRV) and to trigger mobile interventions such as breathing techniques [24]. So-called "mood maps" were used for subjective assessment to correlate HRV measurements with self-perception. In the end, the HRV measurement was discontinued because of the challenges posed by the continuous capturing of sensor data in everyday life and the focus shift to mood maps.

HRV is a physiological parameter that is frequently used for stress recognition. Several studies use HRV to learn a classifier for distinguishing between relaxed states and stressful situations [2, 17, 18]. An approach described in [25] calculates a daily stress score from multiple inputs, among them HRV. The stress-level classifier is trained against a self-assessment questionnaire which the study participants have to fill in several times a day. The correctness of stress-level classification is determined by comparing it with the stress level derived from the questionnaire. Combining all features, an accuracy of $61 \%$ was achieved. Just like in SmartCoping, the authors included an initial learning phase to train their classifier before switching to classification mode. Similarly, user-specific classification models proved superior to classification models learned across all participants.

Skin conductance is another parameter for stress recognition and has been used in a project aimed at recognising stress by means of a wrist sensor which apart from skin conductance also captures movements and mobile phone usage [34]. A self-assessment questionnaire served as the basis for classifying stress.

Some projects focus on developing therapeutic solutions to stress. Interstress, for example, employs biofeedback, meditation and systematic training of coping skills to reduce stress [43]. The projects Monarca, Optimi and Psyche focus on people with mental problems or disorders, which are closely related to stress (see the overview in [33]).

An important application area for stress detection is driver assistance. There are several approaches for detecting driver stress in real-time with HRV playing a major role [26]. In some studies HRV has been supplemented with further parameters such as skin conductance and respiration $[14,32]$ as well as head movements [32]. Another study uses differential skin temperature, measured e.g. at the periphery with sensors and at the face using an infrared camera [45] .

So-called "technostress" has emerged as another interesting and growing application area for stress detection [6]. Whilst until recently usability was largely considered a design issue from the perspective of humancomputer interaction, making the interaction as stressfree as possible has come to be seen as a major goal. Several research projects aim at measuring technostress in a lab setting, comparable to usability tests in usability labs. Others focus on real-time stress measurements during information system usage [46] and investigate how the information system can adapt according to a user's stress level [1]. This requires unobtrusive measuring devices, e.g. a wrist band or a smartwatch. Riedl [31] provides an elaborate survey on the area of technostress.

\section{Innovative Aspects of SmartCoping}

For supporting a user in managing and reducing stress it is not sufficient for an app to display stress symptoms and trigger an alert when these exceed a given threshold. Instead, a more complex logic is required that goes well beyond what is currently offered by stress apps available on the market. In short, SmartCoping comprises the following innovative features:

Automatic user calibration:

A major challenge is posed by the fact that physiological stress parameters vary greatly between individuals depending on age, health status and other factors. This is particularly true for HRV. The SmartCoping app establishes the baseline and stress thresholds for each user via an initial learning phase so that stress measurements and stress warnings are tailored to the individual user.

This approach imposes a minimum burden on the user since there is no need to fill in questionnaires or participate in stress-inducing sessions to calibrate physiological measurements against perceived stress levels.

Relaxation support:

An app for stress detection should not just give stress alerts but help the user relax. The SmartCoping app therefore comprises a biofeedback component which guides the user through breathing exercises while at the same time visualising the actual stress level.

Correlation of recurrent high-stress situations with context data:

Data mining is performed on the recorded stress level history to find out the most stressful situations and how these are related to a user's behavioral patterns or movements so that he or she can develop appropriate avoidance and coping strategies.

Therapeutic effectiveness:

The SmartCoping app has been developed for therapeutic purposes. This is why we have involved medical 
experts right from the start and stress measurement is based on sound medical principles. To collect evidence for the therapeutic efficacy of the app, a randomised controlled open label study with high-risk patients, e.g. patients after alcohol detoxification, burn-out patients, or patients with depression, will be conducted (see Sec.6).

\section{Physiological indicators for stress}

Stress results in the release of adrenaline and, with a certain time delay, cortisol. Cortisol can be measured in the saliva [41], where the change of cortisol level - not the absolute level - is a good indicator for medium-term stress [42]. Short-term stress can best be determined by the adrenaline level in the blood, which requires taking blood samples. Another good indicator of short-term stress is alpha-amylase, which has the advantage that it can be quite easily tested in saliva [27].

Stress causes increased perspiration so stress can be measured via skin conductance, preferably on palms or foot soles [15, 44]. However, skin conductance also increases as a result of regulating body temperature, which diminishes its reliability as an indicator unless it is measured in controlled settings. Facial skin temperature is another indicator for stress [45], as is blood pressure.

Stress levels as well as other emotional states can also be detected from facial expressions $[8,13]$ and from speech via automatic speech analysis [9, 40]. It is difficult, however, to come up with a measurement on a continuous scale of stress levels.

Heart rate variability (HRV) is generally considered a reliable indicator for stress (e.g. [7, 29, 39]). It correlates well with salivary measurements of cortisol and alpha-amylase as has been shown in [22]. Stress reduces the fluctuation of beat-to-beat intervals, leading to lower HRV values, whereas a reduction of stress increases fluctuation and thus corresponds to a higher HRV.

Since SmartCoping aims at measuring stress levels continuously, most of the stress indicating parameters discussed above are not suitable because they either do not allow continuous measurement (cortisol and alpha-amylase in saliva, speech analysis, blood pressure) and/or require special sensor arrangements that are not suitable for everyday use but only in specific circumstances (skin conductance, skin temperature, facial expressions, speech analysis). This leaves only HRV for calculating stress levels in SmartCoping.

\section{Methodological approach}

The SmartCoping system comprises the smartphone app and a backend system (see Fig.1). A body sensor transmits heart rate and interbeat (or RR) intervals in ECG quality with a sampling rate of $1000 \mathrm{~Hz}$ via a bluetooth interface (BLE) to the smartphone app. At present, only chest straps guarantee such quality. Wristbands or smart watches would be much more convenient and less obtrusive for continuous measurement but at present do not achieve the necessary accuracy. However, new devices are under development that are more comfortable to wear than a chest strap and offer ECG quality so that it is a matter of time until this issue is resolved.

The app calculates heart rate and several HRV parameters from the ECG signals (see Sec.5.1) and derives the user's stress level from these measurements (see Sec.5.2). The accelerometer and the GPS receiver built into the smartphone are used to collect context data. The HRV and heart rate data as well as the associated stress level and context data are stored on the backend and can be viewed by the user in the smartphone app or via a web browser. Transmission of data to and its storage on the backend are encrypted.

\subsection{Obtaining physiological stress parameters}

Every 30 seconds over a time window of one minute, the SmartCoping app calculates the average heart rate (which increases with stress) and the following HRV parameters:

- SDNN: standard deviation of RR intervals (i.e., intervals between two heart beats);

- RMSSD: root mean square difference of successive RR intervals in the time frame;

- PNN50: percentage of pairs of adjacent RR intervals differing by more than $50 \mathrm{~ms}$ [4].

We have also experimented with a frequency-based HRV parameter, namely the ratio between low and high frequency spectral powers (LF/HF) [10]. We decided to dismiss this parameter because it typically shows great fluctuations and is therefore not sufficiently reliable for short-term stress detection. Furthermore, breathing often falls into the LF band and can distort HRV measurement unless it is appropriately corrected [5].

Whenever making use of a body sensor one is confronted with the problem of artifacts. Artifacts are either erroneous additional heart beats or beats that dropped due to poor connection of the chest strap to the body. Both kinds of artifacts result in HRV values that are too high and thus distort stress calculation. An effective artifact elimination is therefore quite critical.

SmartCoping eliminates artifacts in three steps: The first step is to reduce the probability of artifacts. Since movement is a major reason for artifacts and also affects HRV, we ignore segments with too much physical activity as detected by the accelerometers in the smartphone or chest strap. The second step is to detect artifacts and 


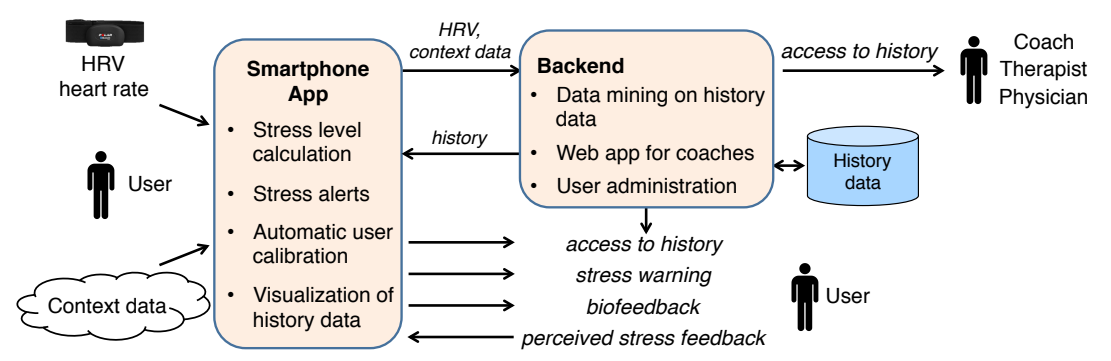

Figure 1. Data flow and architecture of the SmartCoping system

correct them automatically. A filter detects time intervals whose HRV values differ more than an amount $x$ from the surrounding intervals and overrides the measured value with the mean of the surrounding intervals. The value of $x$ ranges between $6 \%$ and $46 \%$, depending on the HRV baseline. In a third step, segments with $3 \%$ or more artifacts are excluded from stress calculation as well as from prompts for stress feedbacks.

\subsection{Automatic calibration to individual users}

Similarly to other projects, we started out by learning classifiers for low, medium and high stress levels using supervised machine learning algorithms. To achieve sufficient accuracy one needs many learning examples, i.e. for each stress level HRV and heart rate measurements together with the associated perceived stress level. Furthermore, while it is relatively straightforward to induce high stress or relaxation in a user and then measure physiological stress parameters, it is much more difficult to obtain intermediate stress levels to measure the parameters for those states. Since SmartCoping aims at recognizing a range of stress levels and not just at distinguishing stress from no stress, obtaining sufficient learning examples would be quite difficult.

Most other projects use a variety of handcrafted features and learn stress level classifiers from large samples of test persons. Because of our focus on HRV, we had to adopt a different approach, namely to acquire the learning input from each user individually because HRV varies greatly across individuals, which has been shown both in lab and real-life settings $[24,25])$. Knowing that typical users would not be prepared to provide lots of feedback on perceived stress levels before they could start using the app, we decided to exploit the fact that rising stress levels correlate with decreasing HRV and increasing heart rate. This allows the SmartCoping app to learn a user-specific stress recognition function with the necessary accuracy from a relatively small number of stress level feedbacks (between 25 and 30). For this purpose, we introduced an initial learning phase during which the app prompts the user for feedback on his or her perceived stress level (see Sec.5.3 for more details).

The highest HRV values coincide with no stress and the lowest values with extreme stress. The naive approach would be to split the interval between minimum and maximum HRV values into three equally large sub-intervals to represent high, medium and low stress levels, respectively. The actual algorithm, however, is more complex because the correlation between perceived stress levels and HRV values is not linear and because we make use of more than just one HRV parameter. The algorithm comprises the following main steps (cf. Algorithm 1):

Line 2: The feedbacks collected during the learning phase result in a set of tuples $\left\langle v_{p}, p s l\right\rangle$ for each HRV parameter $p \in\{s d n n$, rmssd, pnn50 $\}$ where $v_{p}$ is the HRV value measured when the perceived stress level psl has been given by the user (cf. Formula (2)). Higher stress feedbacks correlate with lower HRV values, but this correlation is not perfect since the stress feedbacks are based on subjective perceptions. Therefore, dividing the range of measured HRV values into intervals that correspond to low, medium and high stress typically leads to some misclassified stress feedbacks (cf. Fig.2). The algorithm determines the thresholds between low and medium stress $t l$ and between medium and high stress th in a way that the overall number of misclassifications is minimized. The misclassifications are measured by using the root-mean-square error function that for given thresholds $t l$ and $t h$ adds up the distance of each misclassification to its proper threshold (cf. Formula (1)).

Line 3: Based on the thresholds $t l$ and $t h$, for each HRV parameter $p$ a function slevel $p$ is defined that for a given HRV parameter yields the values 0, 1, 2 for low, medium and high stress, respectively.

Line 6: We need to combine the resulting three stress level functions slevel $p$ into a single function. This is done by computing the weighted mean of the three single slevel $_{p}$ values. The weights are determined according to the predictive accuracy of each HRV parameter in the set of user feedbacks. Our experiments showed that 
the accuracy of the parameters varied greatly between persons which is why the weights were determined for each user individually. We calculate the accuracy $w_{p}$ of an HRV parameter as 1 minus its relative error, i.e. its error divided by the sum of the errors of all parameters.

Line 8: The final stress level is given as the weighted mean of the stress level functions for each HRV parameter. This results in a continuous value between 0 (low stress) and 2 (high stress).

In addition to HRV, heart rate is also used as a stress indicator and needs to be calibrated. This is done in the same manner as with Algorithm 1 except that higher stress levels correspond to higher heart rates whereas it is lower values for HRV. For the sake of simplicity, heart rate calibration is not included in Algorithm 1.

\section{Algorithm 1 Calibrate stress level calculation from HRV}

Input: tuples of HRV values with the associated perceived stress level feedback ( $p s l)$ from the user, i.e. for $p \in$ hrvp $=\{$ sdnn, rmssd, pnn50 $\}:$

$f b_{p}=\left(\left\langle v_{p, 1}, p s l_{1}\right\rangle, \ldots,\left\langle v_{p, 2}, p s l_{2}\right\rangle,\left\langle v_{p, n}, p s l_{n}\right\rangle\right)$

Output: function slevel for calculating the stress level from a given set of HRV values

1: for all $p \in h r v p$ do

2: $\quad$ set thresholds $t l_{p}$ and $t h_{p}$ on the interval of HRV values so that the error of misclassified stress feedbacks in $f b_{p}$ is minimal (see Formula (1)):

$$
\underset{t l_{p}, t h_{p}}{\arg \min }\left(r m s e\left(f b_{p}, t l_{p}, t h_{p}\right)\right)
$$

3: define a function $\operatorname{slevel}_{p}\left(v_{p}\right)$ that gives for an HRV parameter value $v_{p}$ the values $0,1,2$ :

$$
\text { slevel }_{p}\left(v_{p}\right)=\left\{\begin{array}{l}
0: v_{p}>t l_{p} \\
2: v_{p} \leq t h_{p} \\
1: \text { else }
\end{array}\right.
$$

4: end for

5: for all $p \in h r v p$ do

6: calculate the weight for HRV parameter $p$ according to its accuracy:

$$
w_{p} \leftarrow 1-\frac{r m s e\left(f b_{p}, t l_{p}, t h_{p}\right)}{\sum_{p^{\prime} \in h r v p} r m s e\left(f b_{p^{\prime}}, t l_{p^{\prime}}, t h_{p^{\prime}}\right)}
$$

7: end for

8: calculate the overall stress level by taking the weighted mean of all parameters:

$$
\operatorname{slevel}\left(v_{s d n n}, \ldots, v_{p n n 50}\right) \leftarrow \frac{\sum_{p \in h r v p} w_{p} \cdot \operatorname{slevel}_{p}\left(v_{p}\right)}{\sum_{p \in h r v p} w_{p}}
$$

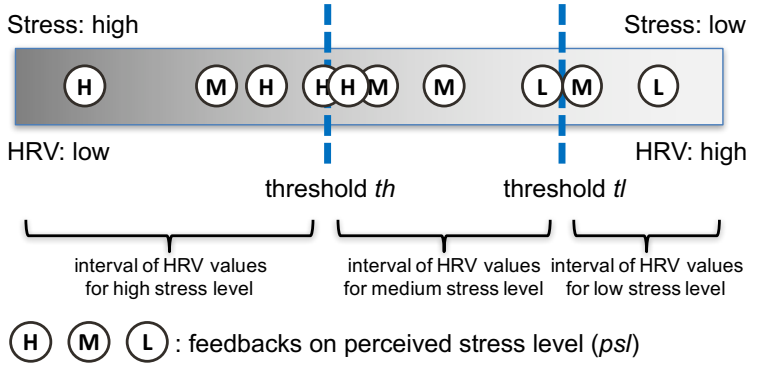

Figure 2. Separating stress level intervals

The formula for determining the error of misclassified stress feedbacks in a set of user feedbacks $f b$ and for thresholds $t l$ and $t h$ is as follows (see also Fig.2):

$$
r m s e(f b, t l, t h)=\sqrt{\frac{\sum_{i=1}^{n}\left(v_{i}-r e f\left(v_{i}, p s l_{i}, t l, t h\right)\right)^{2}}{n}}
$$

where $f b$ is the vector of measured values of a given HRV parameter and associated user feedbacks of low, medium or high stress levels:

$$
f b=\left(\left\langle v_{1}, p s l_{1}\right\rangle, \ldots,\left\langle v_{n}, p s l_{n}\right\rangle\right)
$$

In case of a misclassified HRV value, the function ref returns the threshold to that interval where it correctly belongs. If the HRV value $v$ is correctly classified, the function ref returns that value itself so that it does not count as an error in Formula (1):

$$
r e f(v, p s l, t l, t h)=\left\{\begin{array}{l}
t h: p s l=H \wedge v>t h \\
t h: p s l=M \wedge v \leq t h \\
t l: p s l=M \wedge v>t l \\
t l: p s l=L \wedge v \leq t l \\
v: \text { else }
\end{array}\right.
$$

Physical activity is considered as stress by the body and would therefore trigger stress warnings. To prevent this, the SmartCoping app detects physical activity using the accelerometer built into the smartphone and suppresses warnings during and some time after physical activity. A similar approach is described in [37].

\subsection{Acquiring user feedbacks in the initial learning phase}

The SmartCoping system relies on user feedbacks of perceived stress levels to calibrate the interpretation of HRV and heart rate to the individual user. Studies have shown that stress as experienced by a subject largely coincides with normalised physiological measurements $[12,23]$ so that a user's stress feedbacks can be taken as valid (albeit not exact) stress assessments. 
When starting the SmartCoping app for the first time, the user is presented with a screen as shown in Figure 3 (left). In the initial learning phase, feedback is given with the slider on the right side of the screen. The app maps the slider position to a continuous value between 0 (no stress) and 2 (extreme stress) and divides the interval $[0,2]$ into three equal subintervals which represent low, medium and high stress.

We also experimented with five sub-intervals because we expected that a more fine-grained distinction would yield better stress recognition results. However, it turned out that this led to a higher overall error rate according to Formula (1) than with three intervals. This may be explained by the fact that users can estimate their stress levels only approximately, which is why trying to map their estimates to overly detailed five sub-intervals may cause more inconsistent feedbacks.

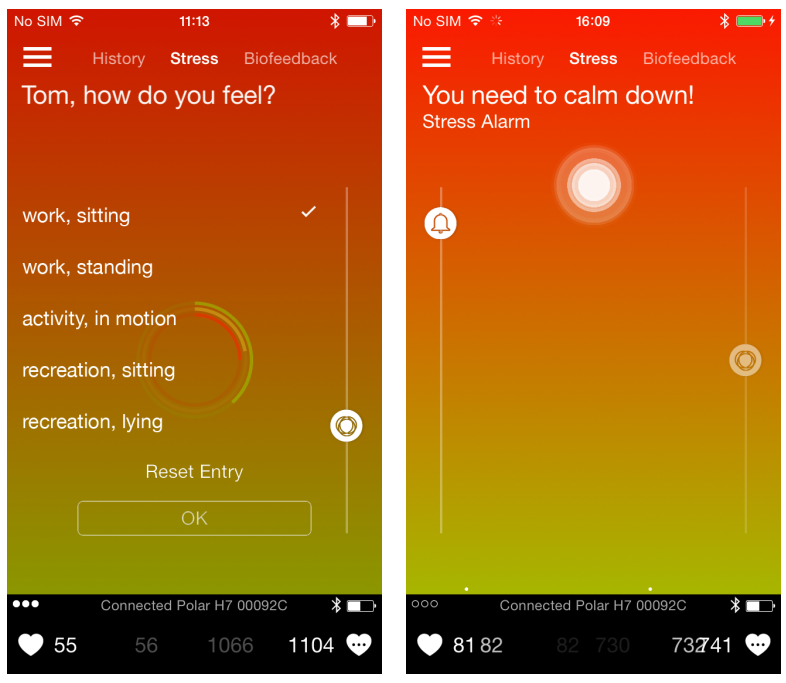

Figure 3. Feedback screen (left); Stress warning screen (right)

When the user gives a stress feedback and the feedback is classified as

- low stress, then the highest HRV value is used

- medium stress, then the mean HRV value is used

- high stress, then the lowest HRV value is used for each HRV parameter over the last three time windows. The heart rate is computed as the mean heart rate in the same time window. Experiments have shown that this way the best correspondence between stress level feedbacks and measured stress parameters is achieved.

The timing of the feedback prompts is controlled by an algorithm which is inspired by the concept of active learning from the machine learning community [35]. Active learning is a form of supervised learning where the learning algorithm asks a user to label a data point. In the case of SmartCoping this means that the prompt- ing algorithm keeps track of feedbacks and the associated HRV values and aims at covering the range of HRV and heart rate values as best as possible to obtain an informative and representative sample. In this way, learning is accelerated so that fewer learning inputs are needed.

The generation of feedback prompts is governed by the following principles:

1. Apart from the prompt generation algorithm, users are free to provide feedback any time, e.g. when they feel particularly stressed or relaxed.

2. During an initial measurement period, no prompts are generated so as to roughly determine the range of heart rate and HRV values.

3. Users can adjust the number of prompts per day so as to prevent them from becoming a nuisance.

4. No prompts for feedbacks are generated during or some time after physical activity - as measured by the accelerometer in the smartphone. This is to avoid contradictory information which would distort stress level calculation.

5. Explore minimum and maximum values:

- Extreme values, i.e. those that exceed previous minimum or maximum values and are not yet covered by a user feedback, generate a prompt.

- If no new maximum or minimum values are encountered for some time, this condition is relaxed so that prompts are generated when only $90 \%$ of previous minimum or maximum values are reached. In other words, the app begins to generate prompts for values slightly below the maximum or slightly above the minimum. Thus the prompting algorithm starts from the extremes and increasingly approaches average values. If the relaxation to $90 \%$ does not lead to new prompts within a given time frame, the condition is further relaxed by another $90 \%$, etc.

6. Explore maximum value changes:

- A decrease or an increase in values within a certain period of measurements (typically three measurements over a time of $90 \mathrm{sec}-$ onds) that is bigger than the amount of change so far observed, prompts a feedback request.

- If no value changes are encountered for some time that are bigger than the ones so far observed, the condition gets relaxed so that prompts are generated when changes reach $90 \%$ of the maximum differences so far encountered. If this does not lead to new prompts, a further relaxation by another $90 \%$ is applied, and so on. 
The coaching phase (cf. Fig.3 (right)) begins once a predefined number of low, medium and high stress level feedbacks have been received so that the user gets stress level readings as soon as possible. The learning phase typically overlaps with the coaching phase and gradually comes to an end once the thresholds $t l$ and $t h$ cease to show any major changes despite additional input (cf. Line 2 of Algorithm 1 and Fig.2). Therefore it is possible that a user might get the occasional prompt even though he or she has already entered the coaching phase.

\subsection{Indication of recurrent high-stress situa- tions}

The primary goal of SmartCoping is not to tell users that they are stressed but improve their self-perception and point them to those situations where they are most likely to feel stress so they may develop appropriate avoidance or coping strategies. These suggestions are generated using data mining techniques performed on the history data stored on the backend. The history data consists of all stress level measurements combined with the following context data:

- Physical activity is measured by an accelerometer in the smartphone or by the heart rate sensor.

- Location is a geographic cell that consists of a range of GPS coordinates. Whenever the user's GPS coordinates fall within that cell, he or she is considered to be at that place. Since a user would find it difficult to interpret GPS coordinates, they are labeled with names of locations relevant to a user, e.g. home, work place, favorite restaurant. For this purpose, the SmartCoping app asks the user to set a label for relevant locations.

- Change of location may be an important indicator for characterizing stress situations.

- Time of day and day of week are also relevant attributes for describing recurrent stress situations.

Other contextual data that may help interpret highstress situations are a user's communication patterns in terms of incoming and outgoing calls, e-mails, text messages and even a semantic analysis of their content. However, this would not only raise data protection and privacy issues but is - depending on the smartphone being used - technically not possible because this kind of data may not be accessible as with iOS, for instance.

Data mining performed on the history data may deliver patterns such as: "Stress level is significantly higher on ..."

... "Tuesday between about 2 pm and 3 pm"

... "weekends spent at the summer house"

... "Friday every other week"

... "Saturday evening at home when mostly sitting for more than 2 hours"

The system learns such patterns by first overlaying the daily (or weekly, fortnightly and monthly) stress level data (recorded every 30 seconds) to obtain an aggregated time segment of one day (or week, fortnight, month). This time segment is divided into $n$ sub-segments, which are shifted in a way that each sub-segment covers a phase of significantly higher average stress level than its neighbouring segments. Finding the proper boundaries for each segment depends on the boundaries of the adjacent segments and is therefore an optimisation problem. We currently tackle this problem by using simulated annealing $[3,19]$. The resulting segmentation is used as input for a further processing step where the description of a time segment is augmented with context data such as location and physical activity when applicable.

\subsection{Biofeedback for reducing stress}

The SmartCoping solution also comprises a biofeedback component to help users relax and reduce stress. The component guides the user through breathing exercises and at the same time visualises the actual stress level by changing the background color from red to green to indicate increasing relaxation. The stress (or relaxation) level is computed in real-time every $30 \mathrm{sec}-$ onds, thus creating a biofeedback loop.

The relaxation effect is due to increasing coherence between breathing and heart rate. To achieve coherence, the biofeedback component offers a pulsating ball as an optional visual pacer for breathing in and out. The timing of the pacer can be set to a default value or be dynamic. In the latter case, based on the last couple of heartbeats the four longest heartbeat intervals are used for timing breathing in, whereas for breathing out, the four shortest heartbeat intervals are used. The number of four heartbeats for the breathing cycle is inspired by yoga breathing (pranayama), where the heartbeat is also used as a pacer for breathing.

The relaxation effect of HRV biofeedback has been well demonstrated in various studies [16, 21, 36, 47]. The Interstress project, which focused on developing therapeutic solutions to stress, also employed biofeedback as a stress reducing technique [43].

\section{Evaluation}

At present, the app is being tested with 35 healthy individuals who have been recruited by the project partners from within their respective organisations. Apart from age, gender and marital status, test persons are also asked to specify their level of education and work status (self-employed vs. employed). After they have 
signed the informed consent form and received detailed information about the SmartCoping project, test persons are shown how to install and use the SmartCoping app. Each test person is given a unique identification number which is also used when they register the app. This allows us to link the data to the respective questionnaires.

The pilot phase lasts four weeks during which test persons have to fill in the German version of the Perceived Stress Questionnaire (PSQ) at the beginning and at the end of the pilot as well as after each week. PSQ has shown to be a valid and economical tool for stress research [11]. The individual assessment of perceived stress is done at three levels (low, medium, high stress). Test persons are also required to fill in the German stress-coping questionnaire SVF 120 at the start and at the end of the testing period. The SVF 120 comprises 120 items and is divided into 20 subtests.

Usability of the app and user satisfaction are also measured, especially patients' judgements of the everyday practicability and convenience of the system. This information will be gathered at the end of the pilot phase in a focus group interview using well-established usability criteria for mobile phone applications.

Subsequently, a randomized-controlled open-label study will be carried out to test the effectiveness of the system for supporting abstinence in alcohol dependent patients during and after in-patient treatment. Perceived effectiveness with regard to the prevention of craving and thus relapse will be measured by the Obsessive Compulsive Drinking Scale (OCDS).

In the clinical study, we will distinguish between two phases: Phase 1, the in-patient treatment phase with instructor-mediated HRV biofeedback training and Phase 2, the post-treatment phase supported by the SmartCoping system. While Phase 1 aims to test the effectiveness of instructor-mediated HRV biofeedback training to increase HRV and reduce craving in alcohol dependent patients during in-patient treatment, Phase 2 will explore the therapeutic benefits and the usability of the SmartCoping system for aftercare support.

For the clinical study, we have submitted a proposal to the ethics commissions responsible for the participating clinics. Once we have received permission, the clinics will start recruiting patients with an ICD-10 diagnosis of alcohol dependence. For data analysis, repeated measures analyses of variance (ANOVA) will be used to test comparisons of the endpoint values against baseline values between groups.

\section{Conclusions}

We have presented the goals, design principles and main algorithms underlying SmartCoping, a system for recognising and managing stress. The overall goal of SmartCoping is to enhance people's self-perception by giving stress alerts. Moreover, the system suggests how a user's stress may be correlated with certain situations, interactions or locations. As opposed to other stress apps, SmartCoping calibrates HRV measures to the user's individual stress levels by means of an initial learning phase where the user provides feedback when prompted by the system. A built-in biofeedback component helps users relax and data-mining performed on the history data supports them in developing stress avoidance and coping strategies by pinpointing the recurrent high-stress situations.

The SmartCoping system targets people who are or feel they are - in danger of chronic stress and highrisk groups such as patients after alcohol detoxification where stress can cause relapse. The app is currently being tested with a group of healthy individuals. Subsequently, a clinical study will validate the app with patients where stress is a major risk factor.

The insights we have gained from SmartCoping are also being transferred to other application areas where stress plays an important role, such as sleep. For example, we are investigating how one's behavior during the day (e.g. physical activity) as captured by body sensors influence sleep architecture and subjective sleep quality. As is the case with stress, the correlations between behavior patterns and sleep vary greatly between individuals which is why our user-specific approach is appropriate here as well. The importance of taking into account individual preferences, attitudes and behavior has been shown time and again in studies related to behavioral change support. In a recent paper [30]. we presented an application framework for a behavioral change support system that comprises various components to adapt system interventions to individual users. Overall we are confident that with our approach, user acceptance as well as the effectiveness of interventions will be improved - be it for coping with stress, with sleep problems or other health issues.

\section{References}

[1] M. Adam, H. Gimpel, A. Maedche, and R. Riedl, "Stress-sensitive adaptive enterprise systems: Theoretical foundations and design blueprint", in Gmunden Retreat on NeuroIS 2014 Proc., F. Davis, R. Riedl, J. vom Brocke, et al., Eds., Gmunden, Austria, 2014.

[2] V. Alexandratos, M. Bulut, and R. Jasinschi, "Mobile real-time arousal detection", in Proc. IEEE Int. Conf. on Acoustics, Speech and Signal Processing (ICASSP), 2014, pp. 4394-4398. 
[3] D. Bertsimas and J. Tsitsiklis, "Simulated annealing”, Statistical Science, vol. 8, no. 1, pp. 10-15, 1993.

[4] K. C. Bilchick and R. D. Berger, "Heart rate variability", Journal of cardiovascular electrophysiology, vol. 17, no. 6, pp. 691-694, 2006.

[5] J. Choi and R. Gutierrez-Osuna, "Removal of respiratory influences from heart rate variability in stress monitoring", Sensors Journal, IEEE, vol. 11, no. 11, pp. 2649-2656, 2011.

[6] A. J. Connolly and A. Bhattacherjee, "Coping with the dynamic process of technostress, appraisal and adaptation", in Proc. 17th Americas Conf. on Information Systems, AMCIS, 2011.

[7] J. P. A. Delaney and D. A. Brodie, "Effects of short-term psychological stress on the time and frequency domains of heart-rate variability", Perceptual and Motor Skills, vol. 91, pp. 514-524, 2000.

[8] D. F. Dinges, R. L. Rider, J. Dorrian, et al., "Optical computer recognition of facial expressions associated with stress induced by performance demands", Aviation, space, and environmental medicine, vol. 76, B172-B182, 2005.

[9] M. El Ayadi, M. S. Kamel, and F. Karray, "Survey on speech emotion recognition: Features, classification schemes, and databases", Pattern Recogn, vol. 44, no. 3, pp. 572-587, 2011.

[10] R. H. Fagard, K. Pardaens, J. A. Staessen, and L. Thijs, "Power spectral analysis of heart rate variability by autoregressive modelling and fast fourier transform: A comparative study", Acta Cardiologica, vol. 53, no. 4, pp. 211-218, 1998.

[11] H. Fliege, M. Rose, P. Arck, et al., "The perceived stress questionnaire (PSQ) reconsidered: Validation and reference values from different clinical and healthy adult samples", Psychosomatic Medicine, vol. 67, no. 1, pp. 78-88, 2005.

[12] T. Föhr, A. Tolvanen, T. Myllymäki, et al., "Subjective stress, objective heart rate variabilitybased stress, and recovery on workdays among overweight and psychologically distressed individuals: A cross-sectional study", Journal of Occupational Medicine and Toxicology, vol. 10, no. 1, p. 39, 2015.

[13] H. Gao, A. Yuce, and J.-P. Thiran, "Detecting emotional stress from facial expressions for driving safety", in Proc. IEEE Int. Conf. on Image Processing, 2014, pp. 5961-5965.

[14] J. A. Healey and R. W. Picard, "Detecting stress during real-world driving tasks using physiological sensors", IEEE Transactions on Intelligent
Transportation Systems, vol. 6, no. 2, pp. 156166, 2005.

[15] T. Huang, J. Xiong, and L. Xuan, "Design and implementation of a wireless healthcare system based on galvanic skin response", in Applied Informatics and Communication, D. Zeng, Ed., Springer, 2011, pp. 337-343.

[16] M. K. Karavidas, P. M. Lehrer, E. Vaschillo, et al., "Preliminary results of an open label study of heart rate variability biofeedback for the treatment of major depression", Applied Psychophysiology and Biofeedback, vol. 32, no. 1, pp. 19-30, 2007.

[17] P. Karthikeyan, M. Murugappan, and S. Yaacob, "Detection of human stress using short-term ECG and HRV signals", Journal of Mechanics in Medicine and Biology, vol. 13, no. 2, 2013.

[18] B. Kaur, J. J. Durek, B. L. O'Kane, et al., "Heart rate variability (HRV): An indicator of stress", in Proc. Independent Component Analyses, Compressive Sampling, Wavelets, Neural Net, Biosystems, and Nanoengineering XII, 2014, pp. 91180V-91180V.

[19] S. Kirkpatrick, C. D. Gelatt, and M. P. Vecchi, "Optimization by simulated annealing", SCIENCE, vol. 220, no. 4598, pp. 671-680, 1983.

[20] A. Legendre and R. Harris, "Exaggerated response to mild stress in rats fed high-fat diet", American Journal of Physiology - Regulatory, Integrative, and Comparative Physiology, vol. 291, no. 5, R1288-1294, 2006.

[21] P. Lehrer, "How does heart rate variability biofeedback work? Resonance, the baroreflex, and other mechanisms", Biofeedback, vol. 41, no. 1, pp. 26-31, 2013.

[22] W. S. Liew, M. Seera, C. K. Loo, et al., "Classifying stress from heart rate variability using salivary biomarkers as reference", IEEE Transactions on Neural Networks and Learning System, 2015.

[23] R. Mandryk and M. Atkins, "A fuzzy physiological approach for continuously modelling emotion during interaction with play environments", Int. Journal of Human-Computer Studies, vol. 6, no. 4, pp. 329-347, 2007.

[24] M. Morris and F. Guilak, "Mobile heart health: Project highlight", IEEE Pervasive Computing, vol. 8, no. 2, pp. 57-61, 2009.

[25] A. Muaremi, B. Arnrich, and G. Tröster, "Towards measuring stress with smartphones and wearable devices during workday and sleep", BioNanoScience, vol. 3, no. 2, pp. 172-183, 2013. 
[26] N. Munla, M. Khalil, A. Shahin, and A. Mourad, "Driver stress level detection using HRV analysis", in Proc. Int. Conf. on Advances in Biomedical Engineering (ICABME), 2015, pp. 61-64.

[27] Y. Noto, T. Sato, M. Kudo, et al., "The relationship between salivary biomarkers and state-trait anxiety inventory score under mental arithmetic stress: A pilot study", Anesthesia \& Analgesia, vol. 101, no. 6, pp. 1873-1876, 2005.

[28] D. Ornish, S. Brown, L. Scherwitz, et al., "Can lifestyle changes reverse coronary heart disease?", Lancet, vol. 336, no. 8708, pp. 129-133, 1990.

[29] R. Orsila, M. Virtanen, T. Luukkaala, et al., "Perceived mental stress and reactions in heart rate variability - a pilot study among employees of an electronics company", Int. Journal of Occupational Safety and Ergonomics, vol. 14, no. 3, pp. 275-283, 2008.

[30] U. Reimer and E. Maier, "An application framework for personalised and adaptive behavioural change support system", in Proc. 2nd Int. Conf. on Information and Communication Technologies for Ageing Well and e-Health (ICT4AWE), 2016.

[31] R. Riedl, "On the biology of technostress: Literature review and research agenda", SIGMIS Database, vol. 44, no. 1, pp. 18-55, 2013.

[32] G. Rigas, Y. Goletsis, P. Bougia, and D. I. Fotiadis, "Towards driver's state recognition on real driving conditions", Int. Journal of Vehicular Technology, vol. 2011, 2011.

[33] G. Riva, R. Baños, C. Botella, et al., "Personal health systems for mental health: The European projects", in Medicine Meets Virtual Reality, J. D. Westwood, S. W. Westwood, L. Felländer-Tsai, et al., Eds., IOS Press, 2011, pp. 496-502.

[34] A. Sano and R. W. Picard, "Stress recognition using wearable sensors and mobile phones", in Proc. Humaine Ass. Conf. on Affective Computing \& Intelligent Interaction, 2013, pp. 671-676.

[35] B. Settles, "From theories to queries: Active learning in practice", Journal of Machine Learning Research: Workshop and Conf. Proc. 16. Workshop on Active Learning and Experimental Design, pp. 1-18, 2011.

[36] M. Siepmann, V. Aykac, J. Unterdörfer, et al., “A pilot study on the effects of heart rate variability biofeedback in patients with depression and in healthy subjects", Appl Psychophysiol Biofeedback, vol. 33, no. 4, pp. 195-201, 2008.

[37] F.-T. Sun, C. Kuo, H.-T. Cheng, et al., "Activityaware mental stress detection using physiological sensors", in Mobile computing, applications, and services, M. Gris and G. Yang, Eds., Springer, 2012, pp. 211-230.

[38] M. Swan, "Health 2050: The realization of personalized medicine through crowdsourcing, the quantified self, and the participatory biocitizen", Journal of Personalized Medicine, vol. 2, no. 3, pp. 93-118, 2012.

[39] G. Tanev, D. B. Saadi, K. Hoppe, and H. B. D. Sorensen, "Classification of acute stress using linear and non-linear heart rate variability analysis derived from sternal ECG", in Proc. 36th Annual Int. Conf. of the IEEE Engineering in Medicine and Biology Society, 2014, pp. 3386-3389.

[40] E. L. van den Broek, F. van der Sluis, and T. Dijkstra, "Cross-validation of bimodal healthrelated stress assessment", Personal and Ubiquitous Comp., vol. 17, no. 2, pp. 215-227, 2013.

[41] M. van Eck, H. Berkhof, N. Nicolson, and J. Sulon, "The effects of perceived stress, traits, mood states, and stressful daily events on salivary cortisol", Psychosomatic Medicine, vol. 58, no. 5, pp. 447-458, 1996.

[42] K. Vedhara, J. Miles, P. Bennett, et al., "An investigation into the relationship between salivary cortisol, stress, anxiety and depression”, Biological psychology, vol. 62, no. 2, pp. 89-96, 2003.

[43] D. Villani, P. Cipresso, A. Gaggioli, and G. Riva, "Positive technology for helping people cope with stress", in Integrating Technology in Positive Psychology Practice, D. Villani, P. Cipresso, A. Gaggioli, and G. Riva, Eds., IGI Global, 2016, pp. 320-347.

[44] M. V. Villarejo, B. G. Zapirain, and A. M. Zorrilla, "A stress sensor based on galvanic skin response (GSR) controlled by ZigBee", Sensors, vol. 12, no. 5, pp. 6075-6101, 2012.

[45] T. Yamakoshi, K. Yamakoshi, S. Tanaka, et al., "Feasibility study on driver's stress detection from differential skin temperature measurement", in Proc. 30th Annual Int. Conf. of the IEEE Engineering in Medicine and Biology Society, 2008, pp. 1076-1079.

[46] J. Zhai, A. B. Barreto, C. Chin, and C. Li, "Realization of stress detection using psychophysiological signals for improvement of human-computer interactions", in Proc. IEEE SoutheastCon, 2005, pp. 415-420.

[47] T. L. Zucker, K. W. Samuelson, F. Muench, et al., "The effects of respiratory sinus arrhythmia biofeedback on heart rate variability and posttraumatic stress disorder symptoms: A pilot study", Applied Psychophysiology and Biofeedback, vol. 34, no. 2, pp. 135-143, 2009. 